\title{
Research and Development of the Engine Combustion System Analysis Platform Based on Cloud Design platform
}

\author{
Yan Wei $^{1, \mathrm{a}}$, Zhang Ruwei ${ }^{2, \mathrm{~b}}$, Tian Congfeng ${ }^{3, \mathrm{c}}$, Wang haikun ${ }^{4, \mathrm{~d}}$ \\ ${ }^{1}$ School of Energy \& Power Engineering, Shan Dong University, Ji Nan, China, 250061 \\ ${ }^{2}$ Jining Coordination Information Technology Co. Ltd, Ji Ning, China, 272073 \\ ${ }^{3}$ Jining Coordination Information Technology Co. Ltd, Ji Ning, China, 272073 \\ ${ }^{4}$ School of Energy \& Power Engineering, Shan Dong University, Ji Nan, China, 250061 \\ ayw65986092@sina.com, bzhang_rw01@163.com, ctiancongfeng@163.com, dwang_haik@126.com
}

Keywords: Cloud-based Design platform; CAE Design; Engine combustion system

\begin{abstract}
In order to shorten the design cycle and to improve the design efficiency of the engine combustion system, a cloud-based design platform of engine combustion system integrating cloud computing, parameterized and modular rapid design is proposed. The overall framework of the engine combustion system analysis cloud platform is built and the architecture and function modules of the cloud-based design platform are analyzed, which are divided into the function management layer, the core function layer and the resource layer. Then, the implementation of finite element cloud analysis is described, including the construction of the whole cloud-based design platform with Python. The feasibility of this cloud-based design platform is verified by the finite element cooperative simulation of a diesel engine combustion system.
\end{abstract}

\section{Introduction}

The engine is the main driving force in many fields such as automobile, agricultural machinery, engineering machinery and national defense equipment. Modern engine research and development involves technologies on combustion, flow, cooling, structure and control. The design of the combustion system is the decisive factor to determine the performance of the engine, and the finite element analysis of the combustion system is the mainstream way of top-down design. However, the cost of finite element analysis is high, and the existing remote finite element analysis method is mainly for single enterprise or a limited number of enterprises, so it is difficult to solve the problem of insufficient capacity on finite element analysis for small and medium enterprises (SMEs) .

As a manufacturing service platform using network and cloud, the cloud manufacturing service platform (CMSP) is a new network manufacturing model, which is based on users' needs to organize online manufacturing resources, and to provide them with a variety of on-demand manufacturing services[1, 2]. Gao pei [3] studied the parametric technology and modularization technology of bridge crane as well as the data storage technology and data management technology of cloud computing. Through these technologies, a bridge crane cloud-based design platform is developed. Ma Chenglong [4] analyzed the key technology of cloud-based design. He studied the architecture and function modules of cloud-based design platform, and focused on the construction of geometric model and remote finite element analysis. $\mathrm{Wu}$ et al. [5] proposed the idea of Cloud-Based Design and Manufacturing (CBDM): CBD and cloud-based manufacturing (CBM) from present available CMfg definitions.

Based on the three-dimensional combustion analysis software Converge, the finite element 
analysis of the engine combustion system is deployed into the cloud-based design platform and integrated with the system. It can solve the problem of uneven resource distribution, difficulties in resource sharing, lack of innovation ability, and poor cooperation between enterprises. It can also achieve the management and sharing of design resources. The platform also improves the design and manufacturing capacity of SMEs.

\section{The Overall Framework of the Engine Combustion System Cloud Design Platform}

In view of the characteristics of different engine combustion systems, the cloud computing technology is introduced into the design process of the diesel engine combustion chamber, and the diesel engine cloud-based design platform which is easy to use for the designers is constructed. The platform architecture includes the function management layer, the core function layer and the resource layer. Using Python to build the entire cloud-based design platform. See Figure 1 for details.

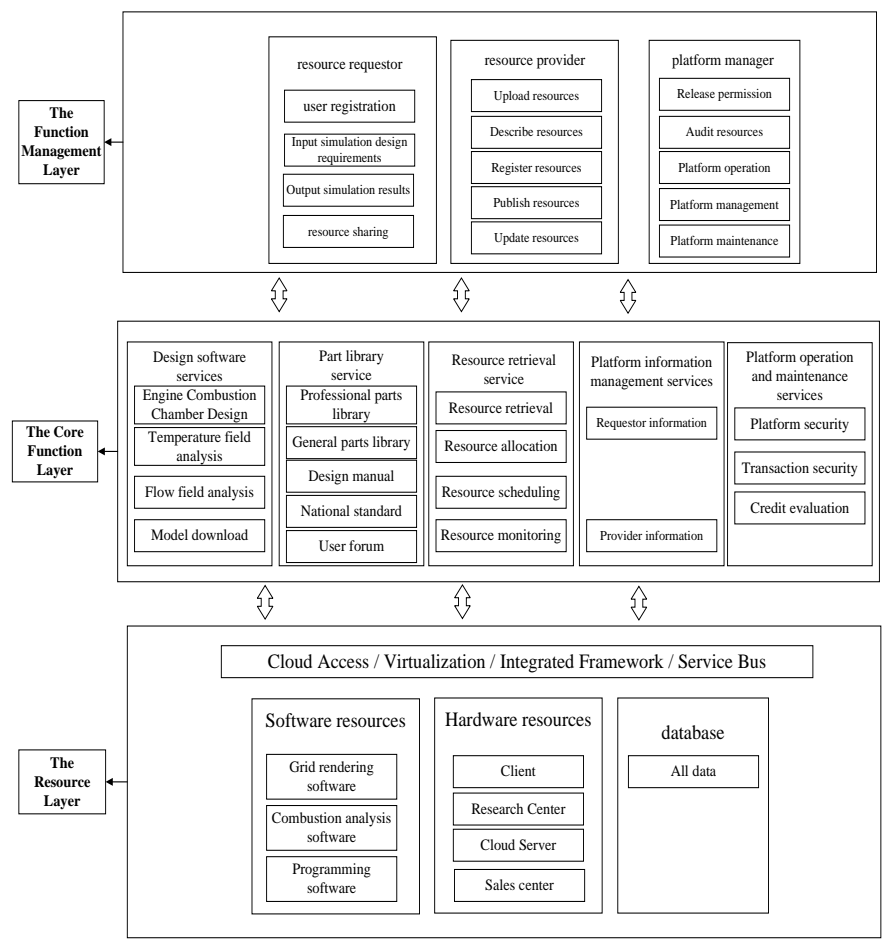

Figure1 the core function framework of cloud-based design

\subsection{The Function Management Layer}

The diesel engine combustion system analysis platform provides users with a variety of services in the form of users accessing to the cloud design platform. The function management layer includes resource requestors, resource providers and platform managers, which is the interface platform to realize the user's human-computer interaction. Users enter the design platform and make an application to the administrator of the function management layer for registration. Users provide the three-dimensional simulation model of the combustion chamber and enter the simulation design requirements. After the simulation calculation is completed, the results will output to the user. 


\subsection{The Core Function Layer}

The core function layer is the most important system function layer of the platform. It provides various core services for resource requestors, resource providers and platform managers. The core services that the platform could provide include simulation software service, engine parts library service, resource retrieval service, information management, platform operation and maintenance service.

The engine combustion system simulation software service provides the following functions: allow the user to provide the three-dimensional model of the engine combustion system, divide grid on the model, set the calculation parameters, analyze the temperature field and flow field, and visualize the calculation results. The user can also download the generated model to the local through the download function on the interface.

\subsection{The Resource Layer}

The main function of the resource layer is to save, manage and use the engine combustion system simulation design virtual resources through the cloud server. The hardware resources provide the actual design resources during the entire simulation design cycle, and the physical design resources include the service clusters needed during the design process. Software resources include a variety of three-dimensional modeling software, simulation software, visualization software and programming software. The resource provider stores the engine design virtual resources (design software, parts library, design manuals, and national standards etc.) into the cloud server's database.

\subsection{The process of virtualizing the design resource}

Currently, most numerical simulation are realized by commercial software. As for combustion simulation, Converge software has got a wide application. In the platform, we try to use the Python to achieve the solving process's seamless access to Converge software. The main body of the program includes three class: ExpObject, Combust_Object and ModelEngine_FormationObject. The ExpObject class completes the modeling process of experiment-related content and belongs to the experimental data, which can be divided into: 0-dimensional bench measurement data, 1-dimensional (time dimension) transient heat release rate, cumulative heat release rate, in-cylinder pressure data, and injection rate curve. The Combust_Object class completes the modeling process of the relevant content in numerical simulation. From a broader perspective, this class is a numerical simulation class, and then accomplishes specific combustion simulation class through implementation. ModelEngine_FormationObject class completes the modeling process of the numerical simulation results analysis. The main variable of the class includes the list storing all case names, the list storing all cases, and the list storing analysis results.

\section{Implementation of Cloud Design process}

\subsection{Introduction to simulation model}

The study object in this paper is a diesel engine. To simulate the combustion, the three-dimensional grid model of the combustion chamber needs to be obtained, and we need to deal 
with the geometric structure of the 3D model. First we need to import the geometric model drawn in Pro / E into the professional CAE software Hypermesh to do geometric cleaning and meshing. After geometrical cleaning, the format of the model needs to be converted into the one which Converge can recognize.

\subsection{Display of the calculation results}

The calculation results are shown in Fig. 2, and the cross section near the lower surface of the fire surface is selected as the study object of the combustion chamber temperature field. In the figure it can be seen the oil has started burning and the high temperature area has generated at $2^{\circ} \mathrm{CA}$ BTDC. At $10^{\circ} \mathrm{CA}$ ATDC the high temperature region has crossed the radial center position, and the combustion range in the individual position has increased which can be seen in the radial section. At $30^{\circ}$ CA ATDC, the combustion has been around the combustion chamber, and there is a significant increase of high temperature region, indicating that the combustion system can meet the actual requirements, it also verifies the effectiveness of the cloud-based design platform.

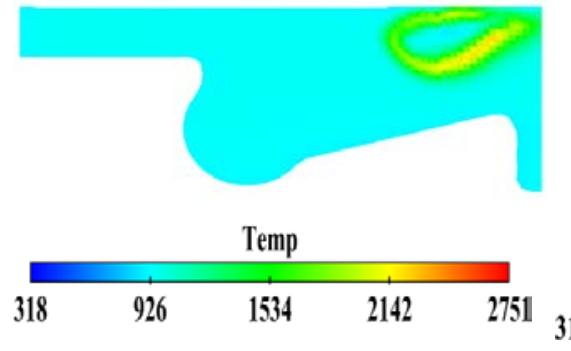

(a) $2^{\circ} \mathrm{CABTDC}$
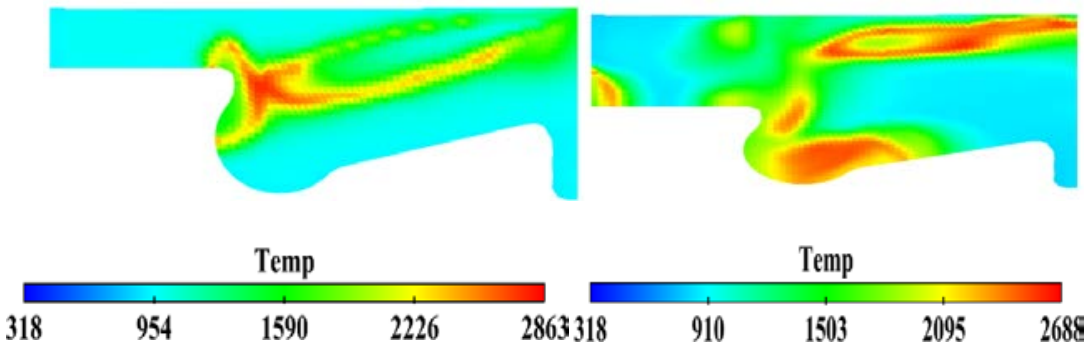

(b) $10^{\circ} \mathrm{CA} \mathrm{ATDC}$ (c) $30{ }^{\circ} \mathrm{CA}$ ATDC

Figure 2 Horizontal distribution of temperature field at rated operating condition

\section{Conclusion}

During the engine combustion system design process, the cost of finite element analysis high, and remote finite element analysis method is mainly for a single enterprise. To solve this problem, based on the cloud manufacturing technology, an engine combustion system cloud-based design platform is proposed. The design flow chart, the design framework and the function module of the cloud service platform are thoroughly studied. The design framework of the engine combustion system is constructed, then the whole cloud design platform is established in Python. The feasibility of the platform is verified by the collaborative simulation of a diesel engine combustion system.

\section{Acknowledgements}

The research was supported by China Torch Program under Grant No. 2015 GH720903.

\section{References}

[1] LI Bo-Hu,L Zhang, L Ren, XD Chai. Further discussion on cloud manufacturing. [J] Computer Integrated Manufacturing Systems, 2011 , 17 (03) :449-457

[2] HK Yin, C Yin, XR Gong, MY Wang, Cloud manufacturing services platform general 
framework and key technology for automobile and motorcycle parts new product development, Computer Integrated Manufacturing Systems, 2013 , 19 (9) :2332-2339

[3] Gao pei. Research on the Construction of Cloud Design Platform of Bridge Crane [M] Taiyuan: North University of China, 2014

[4] Ma Chenglong. Research on the remote analysis service platform based on Cloud Design [M] Hangzhou: Zhejiang University of Technology, 2014

[5] D. Wu, M.J. Greer, D.W. Rosen, D. Schaefer, Cloud manufacturing: strategic vision and state-of-the-art, J. Manuf. Syst. 32 (2013) 564-579. 